\title{
Pérdida inadvertida de un alambre-guía durante canalización de una vía venosa central.
}

Muñoz Corchuelo CM, Gallo Pineda F, Cortiñas Sáenz M.

Hospital Torrecárdenas de Almería.

\section{Resumen}

La cateterización venosa central (CVC) debe ser realizada por profesionales médicos capacitados y/o supervisados en caso de inexperiencia, siguiendo estrictos protocolos de actuación para prevenir incidentes. La incidencia de complicaciones es de hasta un 15\%, algunas potencialmente mortales; estas pueden ser difíciles de evitar, mientras que otras se producen por errores humanos y por lo tanto son susceptibles de intervención y totalmente evitables. Presentamos el caso de una canalización de una vía central con pérdida de alambre-guía de manera inadvertida, descubierta de forma accidental en estudio de imagen por evolución tórpida en postoperatorio, así como la propuesta de un checklist para su prevención.

\section{Introducción}

La cateterización venosa central (CVC) debe ser realizada por profesionales médicos capacitados y/o supervisados en caso de inexperiencia, siguiendo estrictos protocolos de actuación para prevenir incidentes. La incidencia de complicaciones es de hasta un $15 \%$, algunas potencialmente mortales; estas pueden ser difíciles de evitar, mientras que otras se producen por errores humanos y por lo tanto son susceptibles de intervención y totalmente evitables.

Presentamos el caso de una canalización de una vía central con pérdida de alambre-guía de manera inadvertida, descubierta de forma accidental en estudio de imagen por evolución tórpida en postoperatorio, así como la propuesta de un checklist para su prevención.

La inserción de un catéter venoso central en un ser humano se informó por primera vez en 1929 por Werner Forssman (1), quien canalizó su propia aurícula derecha introduciendo un catéter a través de la vena cefálica. Posteriormente, se describieron múltiples accesos para la cateterización de las cavidades cardiacas, pero fue Sven Ivar Seldinger quien revolucionó la medicina al permitir el acceso al sistema venoso central de manera segura y fácilmente accesible, mediante la técnica que lleva su mismo nombre, (2) y que actualmente se considera de elección (3). La CVC es un procedimiento comúnmente realizado en las unidades de cuidados críticos (UCI), en servicios de urgencias y en el quirófano.

Se estima que aproximadamente el $8 \%$ de los pacientes hospitalizados requieren un acceso venoso central durante su estancia. Más de cinco millones de catéteres venosos centrales se insertan al año en los Estados Unidos, con una media de exposición de 15 millones de días con una vía venosa central en un año. (4) 
La incidencia de complicaciones es de hasta un $15 \%$, algunas potencialmente mortales. Existen complicaciones difícilmente evitables, como complicaciones mecánicas, trombóticas e infecciosas, mientras que otras se producen por errores humanos $\mathrm{y}$, por lo tanto, son susceptibles de intervención (tabla 1) (5).

\begin{tabular}{|c|c|c|}
\hline \multicolumn{3}{|c|}{ FACTORES DE RIESGO PARA LA APARICION DE COMPLCACIONES. } \\
\hline FACTOR RIESGO. & MECANICAS-TROMBOTICAS. & INFECCIOSAS. \\
\hline Incidenda & $5-19 \%$ & $5.26 \%$ \\
\hline $\begin{array}{l}\text { Dependientes del } \\
\text { cateter. }\end{array}$ & Trombogenicos & Musilumen \\
\hline $\begin{array}{l}\text { Dependientes del } \\
\text { pesciente. }\end{array}$ & 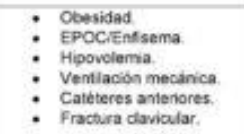 & 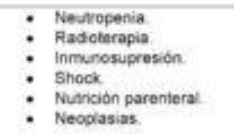 \\
\hline $\begin{array}{l}\text { Dependientes del } \\
\text { lugar de inserción. }\end{array}$ & 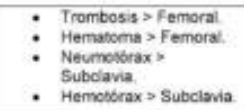 & Femoral > Yugularesubciava \\
\hline $\begin{array}{l}\text { Oependiemles de la } \\
\text { Stcrica }\end{array}$ & $\begin{array}{l}\text { : Punciones multiples } \\
: \text { Difcultad } \\
: \text { Inexpenencia } \\
\text { - Carsacción. } \\
\end{array}$ & $\begin{array}{l}\text { - Puncienes miltiples. } \\
\text { - Alempo permanencia } \\
\text { - Asepsia inadecuada. }\end{array}$ \\
\hline $\begin{array}{l}\text { Tabla } 1 . \\
\text { infecciosas } \\
\text { venosos cen }\end{array}$ & Complicacione & $\begin{array}{c}\text { mecánicas } \\
\text { n de catéter }\end{array}$ \\
\hline
\end{tabular}

Una de estas posibles complicaciones, probablemente poco publicada por temor a litigios, es la pérdida del alambre-guía intravascular durante el procedimiento. Se describe desde hace décadas (6) pero su incidencia está en aumento progresivo en los últimos años. Esta migración del alambre-guía es una complicación rara pero totalmente prevenible y debe ser siempre considerada. Es un evento grave que debe ser comunicado, un ejemplo de lo que en inglés se denomina "never event", término usado a menudo para referirse a un error grave fácilmente prevenible que no debería ocurrir nunca si el equipo asistencial ha implantado las medidas preventivas necesarias. (7)

\section{Descripción del caso}

Varón de 36 años sin antecedentes personales de interés que, tras ser intervenido de apendicectomía laparoscópica, presenta un postoperatorio tórpido por cuadro suboclusivo y distensión de asas de intestino delgado y estómago realizándose reintervención quirúrgica con resección de intestino delgado y divertículo de Meckel. En dicha reintervención se canalizó por anestesiólogo experimentado una vía central de acceso subclavio derecho sin incidencias. A las 96 horas de dicha cirugía se realiza una TAC abominopélvica por clínica de pseudooclusión y se objetiva un alambre-guía con trayecto aparente desde corazón (enrollamiento en aurícula derecha) y descenso a través de la vena cava inferior y presencia de su extremo distal en vena renal derecha. (Figura 1, 2 y 3 ).

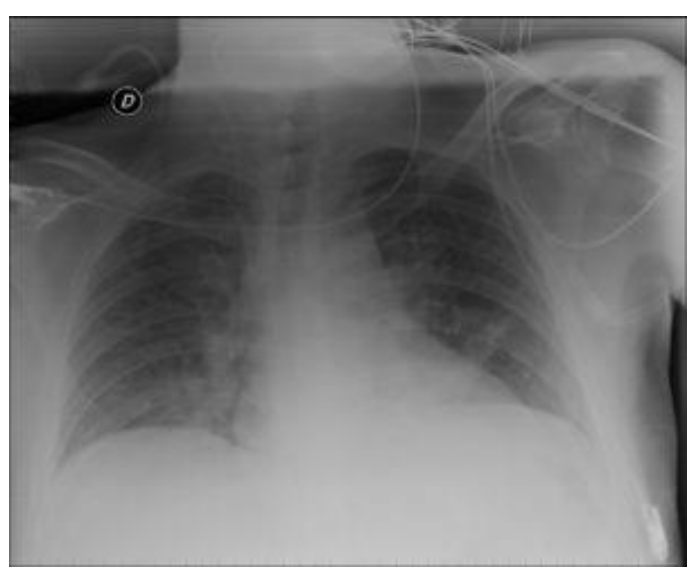

Imagen 1. Alambre guía metálica en radiografía antero-posterior de tórax.

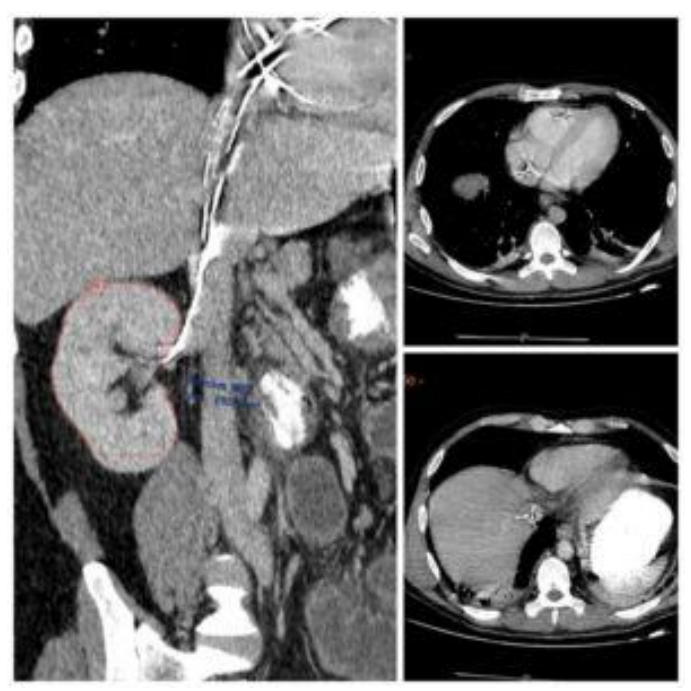

Imagen 2. TAC Abdomino-Pélvico donde se objetiva alambre-guía en aurícula y descenso a través de la vena cava inferior con presencia de su extremidad distal en vena renal derecha. 

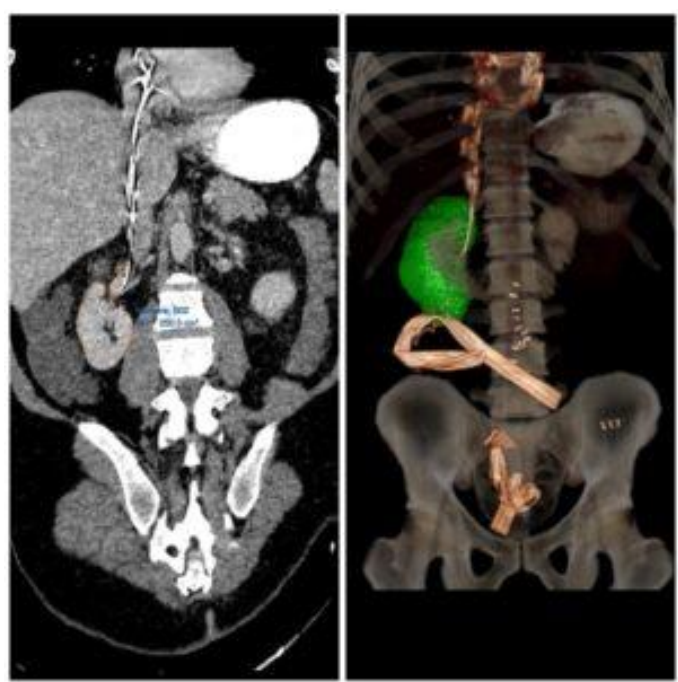

Imagen 3. TAC multidetector de abdomen con contraste y reconstrucción $3 \mathrm{D}$ con realce de órganos (riñón derecho) y volumétrica de esqueleto.

El paciente es trasladado a su centro hospitalario de referencia en cirugía vascular para extracción de la guía con carácter de urgencia por vía femoral, que se logra sin incidencias.

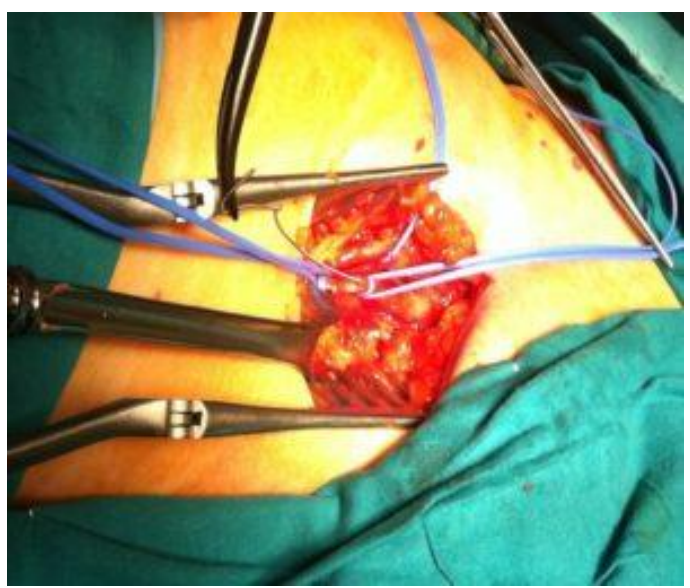

Imagen 4. Extracción de alambre guía por vena femoral externa derecha.

\section{Discusión}

La incidencia de pérdida inadvertida de un alambre-guía durante la CVC es desconocida, pero no es tan infrecuente como se puede creer, ya que se han publicado cerca de un centenar de casos (8). En la literatura revisada, publicada a partir de 2005 se objetiva un incremento importante de los casos reportados de dicha complicación, posiblemente relacionado con la mayor tendencia que existe actualmente a publicar errores/efectos secundarios para análisis del fallo.

En EEUU, esta complicación se incluye dentro de los 27 incidentes graves de declaración obligatoria del National Quality Forum. Estos casos son estudiados posteriormente por los servicios de salud, con la intención de identificar errores predisponentes a dicho incidente e implementar mecanismos que traten de reducir su incidencia. (9), (10). En este sentido, el "checklist" propuesto en la tabla 2 recoge las acciones que deben llevarse a cabo antes, durante y tras cada procedimiento para prevenir este incidente y diagnosticarlo precozmente en caso de que se produzca. (Tabla 2).

Dentro de los principales factores predisponentes para esta complicación, la falta de atención es el factor de riesgo más frecuentemente publicado. Algunas de las situaciones recogidas responsables de esta distracción incluyen: la atención de otros pacientes simultáneamente, cirugía simultánea, atención a enfermería, inestabilidad hemodinámica del paciente $\mathrm{y}$ realización de otras técnicas al mismo tiempo (v.g. un estudio ecocardiográfico).

La presencia de personal excesivo durante el procedimiento también se engloba en este enumerado de factores predisponentes. Otro factor importante es la inexperiencia y la falta de supervisión del operador. Aunque a menudo en los estudios publicados al respecto no recogen estos aspectos, algunas series reflejan que la incidencia de pérdida de alambre guía es mayor cuando la CVC es realizada por personal sin experiencia previa o por médicos residentes, llegando hasta en un $60 \%$ la ausencia de supervisión de estos últimos (7). Se desconoce el número de $\mathrm{CVC}$ que debe realizar un 
operador cada año para mantener sus habilidades o considerarlo personal entrenado, pero si hay varios estudios que muestran mayor tasa de éxito con menores complicaciones en la $\mathrm{CVC}$ en operadores que han insertado más de 50 catéteres. La inserción dificultosa y el número de intentos de punción también se relacionan con la probabilidad de complicaciones, habiéndose demostrado una mayor incidencia cuando se realizan tres $\mathrm{o}$ más intentos $\mathrm{de}$ canalización.

En nuestro caso, la CVC fue realizada por un anestesiólogo con amplia experiencia en dicho procedimiento $\mathrm{y}$ sin reportar dificultad durante la inserción, siendo la distracción un factor probable contribuyente.

Además de reconocer los factores de riesgo anteriormente mencionados, otro punto crucial es la temprana detección del evento para su pronta resolución. Para ello, es esencial la realización de una radiografía de tórax anteroposterior (AP) tras finalizar la técnica, lo cual permite no solo descartar una mal posición del catéter, sino también otras complicaciones como hemoneumotórax, hematomas, etc.

Solo un $25 \%$ de los casos de pérdida del alambre-guía se detecta en el momento de la CVC, al no encontrarse este en la bandeja; hasta en un 30\% de los casos el alambre-guía permanece durante largos periodos de tiempo en el paciente, bien por falta de un estudio radiológico de control, o bien por no identificarse en el mismo, ya sea porque este pasa inadvertido, por no revisar la imagen o por la migración del alambre-guía a vena cava inferior (localización más frecuente) o vena femoral. Para mejorar la detección radiológica de la guía en el momento en que se realiza el control radiológico, es importante retirar del campo los cables del electrocardiograma y cualquier elemento que interfiera con la calidad de la imagen, así como una revisión cuidadosa de la misma. En caso de duda, una radiografía de tórax lateral o la visualización ecográfica puede ser de utilidad.

Otra técnica que ha demostrado ser de utilidad en la detección temprana de canalización arterial errónea y mal posicionamiento de alambre-guía, es la CVC guiada por ultrasonido, que permite su diagnóstico previo a dilatación y colocación del catéter, reduciendo así el tiempo, número de punciones y el riesgo de complicaciones asociadas (11), (12).

Los signos de sospecha de esta complicación son la presencia de una resistencia a la inyección de fluidos a través de la luz distal y/o reflujo venoso deficiente de esta luz distal, que se corrobora con la ausencia del alambre guía en el equipo y su visualización en el control radiológico (7).

Se han publicado diversas complicaciones relacionadas con la pérdida del alambre-guía. Algunas de ellas aparecen de forma temprana, como las arritmias cardiacas, trombosis, embolia pulmonar o infarto cerebral, mientras que otras pueden aparecer pasados meses o incluso años, como endocarditis, sepsis, perforación cardiaca, de grandes vasos sanguíneos perforación o de otros órganos. Existen dos casos publicados de taponamiento cardiaco por perforación de la aurícula y ventrículo derechos por el propio alambre-guía (6); en estos casos, una pericardiocentesis puede solventar el cuadro (13).

No obstante, en la mayoría de casos, la pérdida de alambre guía suele ser asintomática y a menudo se encuentra incidentalmente. 
Tras la identificación del alambre-guía, la extracción por vía percutánea es el método de elección, relegando la extracción quirúrgica para aquellos casos para los que no sea posible, en caso de que sea necesario retrasar el procedimiento por otros motivos, los pacientes deben recibir anticoagulación para prevenir posibles complicaciones embolicas (14).

En aquellos casos en los que la guía se encuentre adherida a la pared del vaso, presente una localización dificultosa para su extracción o la guía se encuentre fragmentada, la extracción supone mayor riego que beneficio, por lo que se mantendrá el alambre-guía dentro del vaso.

\section{Conclusiones}

La pérdida del alambre-guía es una complicación que siempre debemos tener presente y para evitarla, debe mantenerse visible en todo momento. Esta regla es capital para evitar complicaciones asociadas a la entrada de dicha guía en la cámara cardiaca, como problemas de conducción $\mathrm{y}$ arritmias.

Se hace necesario un reporte global de dicho incidente para ayudar al análisis e identificación de los factores predisponentes a este error. $\mathrm{La}$ elaboración de un "checklist" que acompañe a cada técnica es una medida recomendable para una correcta prevención.

La falta de experiencia y de supervisión en caso de personal no experimentado/entrenado son en buena parte responsables de este incidente. El entrenamiento mediante la simulación con maniquíes durante la formación en la práctica médica puede contribuir a la adquisición de habilidad en la técnica de inserción de CVC, sus pasos a seguir, su correcta colocación y comprobación.

\section{Bibliografía}

(1) Seldinger SI. Catheter replacement of the needle in percutaneous arteriography - a new technique. Acta Radiol 1953; 39:368-376. (Pubmed)

(2) Higgs ZC, Macafee DA, Braithwaite BD, Maxwell-Armstrong CA. The Seldinger technique: 50 years on. Lancet 2005; 366 (9494): 1407-1409. ( PubMed)

(3) Hernández MA, Álvarez C, Pérez MA. Complicaciones de la canalización de una vía venosa central. Rev Clin Esp 2006; 206(1):5053. (HTML)

(4) CDC Guidelines for the Prevention of Intravascular Catheter-Related Infections, 2011 http://stacks.cdc.gov/view/cdc/5916/ (Accessed on February 06, 2014). (DF)

(5) McGee DC, Gould MK. Preventing complications of central venous catheterization. N Engl J Med 2003; 348:1123-1133. ( (

(6) Blake PG, Uldall R. Cardiac perforation by a guide wire during subclavian catheter insertion. Int J Artif Organs. 1989; 12:111-113.

(7) Pokharel K, Biswas BK, Tripathi M, Subedi A. Missed Central Venous Guide Wires: A Systematic Analysis of Published Case Reports. Crit Care Med 2015; 43(8):1745-1756. (PubMed)

(8) Gordon PC, Linton DM. The missing wire a complication of central venous catheterization. Anaesth Intensive Care 1992; 20:77-79.

(9) Larizgoitia I, Bouesseau MC, Kelley E: WHO efforts to promote reporting of adverse events and global learning. J Public Health Res 2013; 1;2(3):e29. (PubMed) (HTML) (DF)

(10) National Quality Forum (NQF): Serious Reportable Events in Healthcare - 2011 Update: A Consensus Report. Washington, DC, NQF, 2011. (․ㅐML)

(11) Brass P, Hellmich M, Kolodziej L, Schick G, Smith AF. Ultrasound guidance versus anatomical landmarks for subclavian or femoral vein catheterization. Cochrane Database of Systematic Reviews 2015, Issue 1. Art. No.:

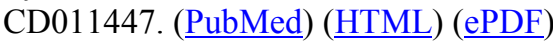


(12) Bedel J, Vallée F, Mari A, Riu B, Planquette $B$, Geeraerts $T$ et al. Guidewire localization by transthoracic echocardiography during central venous catheter insertion: a periprocedural method to evaluate catheter placement. Intensive Care Med 2013; 39:1932 1937. ( PubMed)

(13) Pérez-Díez D, Salgado-Fernández J, Vázquez-González $\mathrm{N}$, et al: Images in cardiovascular medicine. Percutaneous retrieval of a lost guidewire that caused cardiac tamponade. Circulation 2007; 115:e629-e631.

(14) Blinc A, Sabovic M, Bozic M: Low-grade haemostatic activation and increased brin turnover due to a retained central venous guide wire, found accidentally after eight years. Thromb Haemost 2006; 96:852-853. (HTML)

Correspondencia al autor

Carmen María Muñoz Corchuelo carmenmu88@gmail.com

MIR Anestesiología y Reanimación. Hospital Torrecárdenas de Almería.

Aceptado para blog en mayo de 2018. 